SilverStar Förderpreis 2012

\title{
Graswurzelprojekte für Diabetespatienten ausgezeichnet
}

- Zum zweiten Mal wurde der von BerlinChemie gestiftete SilverStar Förderpreis verliehen mit dem Ziel, Initiativen zu fördern, die Patienten mit Diabetes im Alltag unterstützen (www.silverstar-preis.de). Beworben haben sich über fünfzig Projektgruppen. Die Förderanträge wurden von einer Jury bewertet, der u. a. der Diabetologe Prof. Rüdiger Petzoldt, Bad Oeynhausen, und der Geriater Prof. Cornel Sieber, Nürnberg, angehörten.

Der erste Preis (12500 Euro) ging an das Projekt "Gemeindeschwester Muschenheim". Die Bürger von Muschenheim im Städtchen Lich haben einen Verein gegründet, der über Mitgliedsbeiträge zwei Gemeindeschwestern finanziert. Sie kümmern sich in Kooperation mit den jeweiligen Ärzten zu festgelegten Sprechzeiten um die Betreuung älterer Menschen, speziell Diabetespatienten. Zu den Aufgaben der Gemeindeschwestern gehören Therapiekontrollen und Blutzuckermessung, aber auch Schulungen, persönliche Zuwendung und das Lösen von Alltagsproblemen.

Der zweite Preis (7500 Euro) ging an das Projekt Pro Edith am Marienkrankenhaus in Schwerte. Dort wurde ein fachabteilungsübergreifendes Diabetesteam gebildet. Es begleitet Patienten mit der Nebendiagnose Diabetes während des stationären Aufenthalts und übernimmt koordinierende Funktionen beim Übergang in die ambulante Betreuung.

Mehr als 50 Bewerbungen

\section{„Beeindruckende Zahl und Qualität der Versorgungsprojekte"}

\begin{abstract}
Über die Herausforderungen der geriatrischen Diabetiker-Betreuung sowie die Besonderheiten der diesjährigen Preisverleihung unterhielt sich SpringerMedizin mit dem SilverStar-Jury-Mitglied Prof. Cornel Sieber, Chefarzt der Med. Klinik 2, Universität Nürnberg-Erlangen.
\end{abstract}

? Herr Prof. Sieber, welches sind die Herausforderungen in der Diabetologie beim alten, geriatrischen Patienten?

Sieber: In der Geriatrie haben wir es häufig mit Patienten zu tun, die mehrere Krankheiten aufweisen und viele Medikamente einnehmen. Der Diabetes ist bei diesen Patienten eine der häufigsten Komorbiditäten. In unserer Klinik beträgt das Durchschnittsalter 83 Jahre und etwa 25\% der Patienten haben einen Diabetes mellitus.

Die Herausforderung für den Geriater besteht in der Regel darin, eine gewisse Priorisierung innerhalb der Multimorbidität vorzunehmen und jene Störungen zu behandeln, welche die Funktionalität des Patienten einzuschrän- ken drohen. Der Diabetes nimmt hier einen hohen Stellenwert ein, weil er zu Komplikationen in mehreren Organsystemen führen kann, die den Patienten deutlich behindern.

? Können wir die Ergebnisse aus randomisierten kontrollierten Studien ohne Weiteres auf geriatrische Patienten übertragen?

Sieber: Im Vergleich zu anderen Krankheiten ist die Datenlage beim Diabetes im Alter recht gut. Wir wissen, dass es sich lohnt, den Stoffwechsel des Diabetikers auch im Alter gut einzustellen, wobei man bei den Zielwerten etwas großzügiger ist. Denn im Alter sollten Hypoglykämien aus vielen Gründen unbedingt vermieden werden.

? Wie beurteilen Sie das Projekt SilverStar? Sieber: Mir hat der SilverStar von Anfang an sehr gut gefallen, weil hier Projekte gefördert werden, die klar die Versorgung älterer Menschen mit Diabetes im Fokus haben. Wir haben in Deutschland das Problem, dass die Versorgungsforschung nicht den gleichen
Ebenfalls mit einem zweiten Preis $(5000$ Euro) ausgezeichnet wurde eine Initiative des Agaplesion Bethesda Krankenhauses Stuttgart. In dieser Klinik erhalten Diabetespatienten noch während des stationären Aufenthalts eine Essensunterlage, auf der der richtige Umgang mit der Insulintherapie grafisch und in Worten dargestellt ist. Diese Unterlage können die Patienten später mit nach Hause nehmen und haben so die wichtigsten Informationen immer zur Hand.

\footnotetext{
- Philipp Grätzel von Grätz

Quelle: Preisverleihung auf der Herbsttagung der Deutschen Diabetes Gesellschaft, Berlin, November 2012 (Veranstalter: Berlin-Chemie)
}

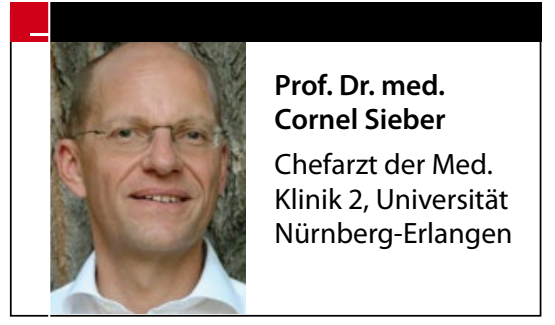

Stellenwert innehat wie in vielen anderen europäischen Ländern, und entsprechend Versorgungstrukturen weniger untersucht und gefördert werden. Das SilverStar-Projekt füllt hier eine Lücke, in dem es Projekte auszeichnet, die multidisziplinär aufgesetzt sind und die eine Praktikabilität im Alltag aufgezeigt haben. Damit hat dieser Preis durchaus einen visionären Charakter.

? Sind Sie überrascht, dass sich mehr als 50 Projekte für den Preis beworben haben? Sieber: Absolut, und dies zeigt, dass es im deutschen Gesundheitssystem viele gibt, die sich mit persönlichem und teilweise ehrenamtlichem Engagement bemühen, die Versorgung von älteren Menschen mit Diabetes zu verbessern. Wenn ich mir die Zahl der Bewerbungen und die Qualität der Projekte ansehe, die sich hier beworben hat, dann beeindruckt mich das schon sehr.

- Interview: Dr. med. Dirk Einecke 